\title{
Assessing Patient Outcomes after Palliative Radiotherapy Using IG-IMRT
}

\author{
Rajiv Samant ${ }^{1,2 *}$, Michael Scopazzi ${ }^{3}$, Kathy Carty $^{2}$ \\ ${ }^{1}$ The Ottawa Hospital Cancer Centre, Ottawa, Canada; ${ }^{2}$ Faculty of Medicine, University of Ottawa, Ottawa, Canada; ${ }^{3}$ Northeastern \\ Ontario Regional Cancer Centre, Sudbury, Canada. \\ Email: *rsamant@ottawahospital.on.ca
}

Received September $7^{\text {th }}$, 2012; revised October $9^{\text {th }}, 2012$; accepted October $20^{\text {th }}, 2012$

\begin{abstract}
Purpose: To assess clinical outcomes after using IG-IMRT for palliation among patients with advanced cancers. Methods: Patients with advanced and/or metastatic cancers were treated on our Tomo-PAL (Tomotherapy ${ }^{\circledR}$-Planning and Administration Linked) protocol using helical TomoTherapy ${ }^{\circledR}$ and evaluated to assess clinical efficacy of treatment as well as to assess side effects. Results: A total of 40 patients were treated to 40 sites from Feb 2007 to May 2009. There were 25 men and 15 women with a median age of 70 years (range 16 -94). Pain and bleeding were the most common symptoms being palliated (80\% and $12.5 \%$ respectively). The dose prescribed ranged from $5-25$ Gy in 1 - 5 fractions. A qualitative improvement in symptoms was documented in $82 \%$ of patients ( $75 \%$ partial relief and $7 \%$ complete relief) and major side effects were not encountered. Conclusions: IG-IMRT can be used for palliation and produces response rates that compare favourably with those reported in the published literature.
\end{abstract}

Keywords: Radiotherapy; Palliation; Symptom Improvement; IG-IMRT

\section{Introduction}

Radiotherapy has long been proven as an effective tool in the palliation of symptoms caused by locally advanced and/or metastatic cancer [1-3]. Approximately 30\% $50 \%$ of a cancer center's radiotherapy workload can consists of palliative treatments, all of which are focused towards managing patients' symptoms which can adversely affect their quality of life [4,5]. Pain is the most common and burdensome symptom experienced by patients with advanced cancer and radiotherapy is very useful for treating this problem [6,7]. A meta-analysis completed in 2007 estimates that the prevalence of pain in patients with cancer at any stage of disease can be in excess of $50 \%$, and in at least one third of the cases the pain is rated to be moderate or severe [8]. The instance of pain is even higher in patients with advanced and/or metastatic cancer.

Though exact numbers are difficult to determine from the published literature, radiotherapy has been estimated to effectively palliate symptoms in $50 \%$ - $80 \%$ of patients with advanced stage disease with relatively little toxicity [9]. An overview of the published literature [1-3,9] suggests palliative radiotherapy response rates in the range of $70 \%-94 \%$ for at least partial relief of metastatic bone

"Corresponding author. pain, $72 \%$ - 86\% for hemoptysis, $60 \%$ - 90\% for superior vena cava obstruction, and $64 \%$ - $73 \%$ spinal cord compression. These studies confirm that palliative radiotherapy plays a very significant role in the treatment of many patients and therefore worthwhile to investigate new approaches for treating these symptoms efficiently and effectively.

A typical palliative radiotherapy treatment plan is usually quite simple and often involves a strategy with either a single direct beam or parallel-opposed-pair technique $[2,10]$. Treatment planning for this process usually begins with the patient undergoing fluoroscopic (conventional) or computerized tomographic (CT) simulation, then the necessary treatment calculations and appropriate plans need to be developed before the patient can have the first treatment. In previously published study from our center [11], it was found that, on average at The Ottawa Hospital (TOH), it took 3 hours for the entire process using conventional fluoroscopic simulation and approximately 3.5 hours for those undergoing CT simulation. The patients typically send a considerable amount of time waiting after the simulation before they can have their treatment. They are usually moved to another area and wait before being brought in for treatment, and at the time of treatment they must be repositioned again on the treatment couch. However, more modern and sophisti- 
cated approaches to plan and deliver radiation are now available $[12,13]$.

We have investigated the potential benefits of using helical TomoTherapy ${ }^{\circledR}$ with StatRT ${ }^{\circledR}$ software in a protocol called Tomo-PAL (Tomotherapy_-Planning and Administration Linked) which integrates a built-in megavolt CT, dedicated inverse treatment planning, adaptive planning tools, and image-guided intensity-modulated radiotherapy (IG-IMRT) dose delivery into a single treatment unit [11]. The entire scan, plan, and treat process can be completed in approximately one hour on this single machine. The advantage is that the patient can remain on the treatment couch and in one room for the duration of the entire process and may only need to be set up once by a team of therapists. The patient does not have to repeatedly move from one area or room to another. This can limit the aggravation of painful symptoms form repeated movement and setups and reduces the number of staff involved ion the treatment. This also allows the patient to feel more comfortable and reduces the amount of time the patient spends in the radiotherapy department. Thus, the end result being a treatment process that is streamlined and more efficient for patients, and reduces their waiting in the radiotherapy department.

Our Tomo-PAL protocol has demonstrated how efficient the entire is purely from a time and resource utilization prospective. However, we hypothesized that palliative radiation treatment with this IG-IMRT approach should also provide clinically significant improvement in patient symptoms which would compare favourably with response rates cited in the literature with traditional approaches without increase in expected acute side effects. We therefore decided to retrospectively review clinical outcomes in patients treated on our previously published Tomo-PAL protocol.

\section{Methods}

After receiving ethics approval, a retrospective chart review was conducted on all patients treated on the TomoPAL protocol for advanced stage cancer. All the data was obtained from both TOH electronic records as well as hard-copy charts. Patients were treated on the TomoPAL protocol from February 2007-May 2009, and all are included in this study.

Data pertaining to symptom relief and acute side effects was extracted from electronic and paper records and entered into a Microsoft Excel spreadsheet. Data was collected at the time of patient treatment as well as for up to 5 follow up appointments. Data collected included diagnosis, presenting symptoms, treatment prescribe, as well as symptom response and side effects from treatment. If there was missing data in any category, it was classified not evaluable.
Once the data was collected the categorical means, maximum values and minimum values where calculated. Finally the data was analyzed to determine if there was any correlation between various aspects of patient, tumour and treatment factors and response rates. To measure the significant of these comparisons, Pearson's Chisquare test was preformed.

\section{Results}

The study population consisted of 40 patients treated on the Tomo-PAL protocol and a total of 40 sites were treated. The median age of patients was 70 years, with a range of 16 - 94 years. There were slightly more male then female patients (62.5\% versus $37.5 \%)$. The most common cancer diagnoses included prostate (22.5\%), bladder (15\%), and breast (10\%). As shown in Figure 1, pain was overwhelmingly the most common symptom (80\%) that patients presented with and for which they received palliative radiotherapy. Of the 40 patients, 32 appeared to have radiotherapy aimed towards pain relief. The causes of pain included the following: compression of vertebral bodies of the spine; bone fracture; pressure caused by bulky disease impinging on nerves; and total destruction of bone by cancer. Five patients (12.5\%) were treated in an effort to stop bleeding and the remaining three patients were given palliative radiotherapy treatment for other symptoms.

Table 1 indicates that the sites of radiotherapy were mainly the axial skeleton (80\%) including the spine, pelvis and sacrum. A small proportion (20\%) of patients had radiotherapy to the abdomen, extremities or lungs. The

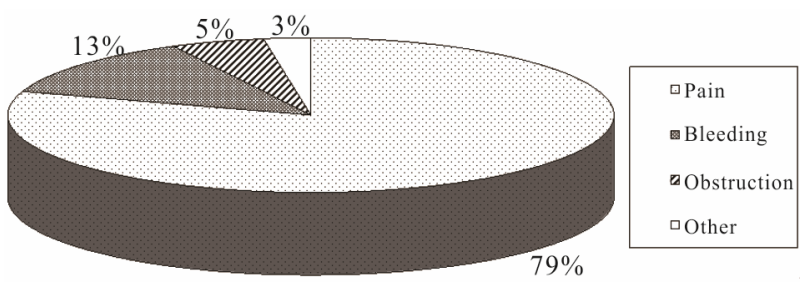

Figure 1. Summary of patient symptoms for which palliative radiotherapy was used.

Table 1. Summary of sites treated with radiation.

\begin{tabular}{ccc}
\hline Site & $\begin{array}{c}\text { Number of patients } \\
\text { treated }\end{array}$ & \% Treated \\
\hline Spine & 15 & $37.5 \%$ \\
Pelvis & 12 & $30 \%$ \\
Sacrum & 5 & $12.5 \%$ \\
Abdomen & 3 & $7.5 \%$ \\
Extremities & 3 & $7.5 \%$ \\
Lung & 2 & $5 \%$ \\
\hline
\end{tabular}


palliative radiotherapy doses prescribed rage from $5 \mathrm{~Gy}$ to $25 \mathrm{~Gy}$ in 1 to 5 fractions. The most commonly prescribed dose fractionation regimen was $8 \mathrm{~Gy}$ in 1 fraction (35\%), followed by $20 \mathrm{~Gy}$ in 5 fractions (22.5\%). These coincide with the two most typical dose fractionation regimens for palliative radiotherapy used at the Ottawa Hospital Cancer Centre. It was found that nearly half of the patients $(42.5 \%)$ had prior radiotherapy treatments to the site being treated using helical TomoTherapy.

Often the severity of the pain experienced by patients can be described through the use of a visual analog scale where the patient rates their pain between 0 and 10. Only 12 of the 40 treated patients (30\%) had visual analog scores recorded in their charts during the initial consultation with the oncologist. Of these patients almost $60 \%$ considered their pain severe (ranging from 8 - 10) with 2 patients considering their pain moderate (ranging from 4 7) and 1 patient had mild pain (scored as three or less). Two patients recorded having no pain on the visual analog scale and they were being treated for other symptoms. Unfortunately follow-up pain scores were not reliably or consistently recorded. Much of the documentation and subsequent evaluation was based on general verbal communication with the patient and oncologist during follow-up appointments and was assessed as qualitative data regarding the current state of their symptoms.

Most patients had follow-up approximately one month post-radiation and then every one to three months afterwards, and the summary of symptom response is shown in Table 2. At the time of first follow-up, 28 of the 40 patients (70\%) had some either qualitative or quantitative documentation of their response within the patient record. Of these 28 documented responses; $75 \%$ had a partial decrease in their symptoms, $7 \%$ had complete pain or symptom relief, $14 \%$ had no change, and $4 \%$ had increasing symptoms after treatment. Table $\mathbf{3}$ shows a summary of responses at the first follow-up visit according to gender, site treated, symptoms, dose and prior radiation. The response rates for pain relief and bleeding were similar and the majority of responses appeared to be sus-

Table 2. Summary of symptom response according to followup (FU) visit.

\begin{tabular}{cccccc}
\hline $\begin{array}{c}\text { Symptom } \\
\text { response }\end{array}$ & FU-1 & FU-2 & FU-3 & FU-4 & FU-5 \\
\hline No change & 4 & 2 & 1 & 3 & 4 \\
Increase & 1 & 4 & 4 & 5 & 4 \\
Decrease & 21 & 12 & 8 & 3 & 2 \\
Complete relief & 2 & 3 & 2 & 2 & 0 \\
$\begin{array}{c}\text { Data not } \\
\text { available }\end{array}$ & 12 & 19 & 25 & 27 & 30 \\
\hline
\end{tabular}

Table 3. Summary of Response vs No Response at first follow-up based on gender, site, symptom, dose, and prior RT (radiation).

\begin{tabular}{|c|c|c|c|}
\hline Variable & Response & No Response & Total \\
\hline \multicolumn{4}{|l|}{ Gender } \\
\hline Male & 14 & 2 & 16 \\
\hline Female & 9 & 3 & 12 \\
\hline \multicolumn{4}{|l|}{ Site } \\
\hline Spine & 13 & 3 & 16 \\
\hline Pelvis & 6 & 1 & 7 \\
\hline Other & 4 & 1 & 5 \\
\hline \multicolumn{4}{|l|}{ Symptom } \\
\hline Pain & 21 & 4 & 25 \\
\hline Other & 2 & 1 & 3 \\
\hline \multicolumn{4}{|l|}{ Dose } \\
\hline $8 \mathrm{~Gy}$ & 7 & 1 & 8 \\
\hline 20 Gy & 9 & 0 & 9 \\
\hline Other & 7 & 4 & 11 \\
\hline \multicolumn{4}{|c|}{ Prior Radiation } \\
\hline Yes & 12 & 0 & 12 \\
\hline No & 11 & 5 & 16 \\
\hline
\end{tabular}

tained at the second follow-up visit which was one to three months later. However, for the subsequent follow-up appointments there was less complete documentation of patient symptoms. Overall response rates were then calculated by determining whether patients were responders or non-responders at the time of follow-up and dividing by the total number of documented entries for that follow-up. The symptom response rate for the first follow-up was approximately $82 \%$ but declined to $71 \%$ and $67 \%$ respectively for the second and third follow-up visits (Table 3).

Unfortunately there was very little documentation recorded in patient charts with respect to experienced side effects. Of the 40 patients treated under the protocol, only 8 had any side effects mentioned in the chart and they generally were quite minor and included nausea and vomiting, diarrhea, fatigue, and pain flare. There were no treatment related complications that resulted in hospitalization or death. The average length of response documented in the study was 25 weeks, with maximum response lasting up to 184 weeks. At the time of analyses $55 \%$ had died. The Pearson Chi-squared test was preformed to determine whether there was a correlation between observed response rates and a number of patient 
and treatment related factors. Only prior radiotherapy appeared to predict for significant symptom improvement at the first follow-up visit with a p-value of less than 0.05 but when we evaluated response rate at the second or subsequent visits the prior radiotherapy did not appear to be statistically correlated with responses. Gender, treatment sites, symptoms and dose were not correlated with response rates

\section{Discussion}

Our Tomo-PAL protocol results suggest that palliative IG-IMRT using helical TomoTherapy with StatRT software provides patients with symptom relief that is at the very least comparable to palliative radiotherapy treatments with more traditional approaches. The response rates for the first three follow-up visits $(82 \%, 71 \%$, and $67 \%$ respectively) compare very favourably with the response rates noted in other published studies [1-3]. They also compare favourably to response rate reported by Lee et al. in their 2009 study [14] evaluating TomoTherapy treatment for pain relief of spinal metastasis ( $76 \%$ response rate). Other studies have also demonstrated high rates of pain relief as well as improvements in quality of life after using TomoTherapy ${ }^{\circledR}$ for the treatment of bone metastases $[15,16]$. Among our patients, by the fourth and fifth visits the response rates drop to $38 \%$ and $20 \%$ but this may be due to documentation issues rather then a true decrease in response rates. Other possibilities include the fact the patient may have out-lived the effects of their palliative treatment or the patient may have developed new symptoms within un-irradiated sites. The most commonly used fractionation regimens prescribed on the Tomo-PAL protocol were similar to those with the standard traditional approaches (8 Gy in a single fraction and 20 Gy in 5 fractions). Previous studies have suggested that there is no significant difference in palliative response between these two dose fractionation regimens when used for symptomatic bony pain [17]. Therefore it appears quite appropriate to prescribe $8 \mathrm{~Gy}$ in a single fraction whenever possible. This would be far more convenient for patient and minimizes the amount of travel and repeated daily visits. It also would reduce the burden on the radiotherapy treatment machines.

Patients who receive palliative radiotherapy often have multiple sites treated and therefore it is not surprising that nearly half of the patient were receiving radiotherapy to or near an area treated previously. Interestingly there was a statistically significant improvement in response rates when patient were retreated to an area, although this lost significance subsequent visits. This may partly be due to the fact that patients were selected for retreatment to an area only if they had a good response previously.

Our study suggests that at least on the Tomo-PAL protocol, pain was the most common symptom being treated with palliative radiotherapy although published literature would suggest this is also the case elsewhere. Locally advanced metastatic cancer results in a high prevalence of bony metastasis especially to areas such as the spine, pelvis and sacrum. Fortunately these can be well palliated with radiotherapy.

Our study does have a number of limitations despite the fact it shows good response rates. First of all pain is sometimes difficult to evaluate if quantitative scales are not used and majority of patients did not have visual analog pain scores documented. Qualitative description of pain are still of value but far more subjective and likely inconsistent in terms of variability between observers. They would also be subject to physician bias as they are recorded by the health care provider instead of the patient. Also, our patient population (40) was relatively small and the amount of follow-up documentation with subsequent visits was quite limited. This makes it difficult to perform statistical analyses and or show any statistical correlations. Finally, it was not possible with our data to accurately evaluate acute side effects caused by radiotherapy. This was because there was little documentation found in the patients and essentially it was entirely quantitative. Helical TomoTherapy ${ }^{\circledR}$ has previously been shown to reduce toxicity and improve quality of life in the palliative setting [18], and it is reasonable to assume that most of the side effects among our patients were mild to moderate since the expectation would be that severe side effects would be more likely to be documented in the patient records. However, this is purely conjecture basis on assumptions related to routine practice of patient documentation. One could assume that with IMRT there could in fact be less severe side effects due to reduced radiotherapy doses to adjacent normal structures but we were unable to demonstrate this. More consistent use of symptom assessment scales such as The Edmonton Symptom Assessment System (ESAS) could help in the future to document changes in patients' symptoms between visits. Better documentation and quantification of toxicities would also be useful to assess side effects. This could be considered for future studies since symptom improvement as well as side effect treatment contribute to patient's quality of life.

\section{Conclusion}

Palliative radiation therapy forms a large proportion of the work load of many radiotherapy departments and delivering IG-IMRT using TomoTherapy ${ }^{\circledR}$ provides an efficient alternative to traditional palliative radiotherapy techniques. The ability to scan, plan, and treat patients all on a single unit in a single appointment is very convenient for patients, and this retrospective study suggests that response rates compare vary favourably (over $80 \%$ within one month) with those in the published literature 
with more traditional approaches. There was also no difference in response rates related to the site or symptom being treated or the dose fractionation regimen used. However due to the small sample size and lack of adequate documentation is not possible to make a definitive conclusion regarding the side effects related to this approach.

\section{Acknowledgements}

Then authors would like to thank the Ottawa Cancer Foundation for financial support of this study and Ms. Betty McAndrew for her administrative assistance.

\section{REFERENCES}

[1] J. P. Ciezki, S. Komurcu and R. M. Macklis, "Palliative Radiotherapy," Seminars in Oncology, Vol. 27, No. 1, 2000, pp. 90-93.

[2] D. Hoegler, "Radiotherapy for Palliation of Symptoms in Incurable Cancer," Current Problems in Cancer, Vol. 21, No. 3, 1997, pp. 129-183. doi:10.1016/S0147-0272(97)80004-9

[3] M. Ashby, "The Role of Radiotherapy in Palliative Care," Journal of Pain and Symptom Management, Vol. 6, No. 6, 1991, pp. 380-388. doi:10.1016/0885-3924(91)90030-8

[4] G. Stevens and I. Firth, "Clinical Audit in Radiation Oncology: Results from One Centre,” Australasian Radiology, Vol. 40, No. 1, 1996, pp. 47-54. doi:10.1111/j.1440-1673.1996.tb00345.x

[5] M. V. Williams, N. D. James, E. T. Summers, A. Barrett and D. V. Ash, "On Behalf of the Audit Sub-Committee, Faculty of Clinical Oncology and the Royal College of Radiologists National Survey of Radiotherapy Fractionation Practice in 2003,” Clinical Oncology (Royal College of Radiologists), Vol. 18, No. 1, 2006, pp. 3-14. doi:10.1016/j.clon.2005.10.002

[6] R. Andrade, et al., "A Simple and Effective Daily Pain Management Method for Patients Receiving Radiation Therapy for Painful Bone Metastasis,” International Journal of Radiation Oncology*Biology*Physics, Vol. 78, No. 3, 2010, pp. 855-859.

[7] L. Vakeat and T. Boterberg, "Pain Control by Ionizing Radiation of Bone Metastasis," International Journal of Developmental Biology, Vol. 48, No. 5-6, 2004, pp. 599606. doi:10.1387/ijdb.041817lv
[8] M. Van den Beuken-van Everdingen, et al., "Prevalence of Pain in Patients with Cancer: A Systematic Review of the past 40 Years," Annals of Oncology, Vol. 18, No. 9, 2007, pp. 1437-1449. doi:10.1093/annonc/mdm056

[9] R. Samant and A. C. Gooi, "Radiotherapy Basics for Family Physicians: Potent Tool for Symptom Relief," Canadian Family Physician, Vol. 51, No. 11, 2005, pp. 1496-1501.

[10] A. Konski, S. Feigenberg and E. Chow, "Palliative Radiation Therapy," Seminars in Oncology, Vol. 32, No. 2, 2005, pp. 156-164. doi:10.1053/j.seminoncol.2004.11.013

[11] M. MacPherson, et al., "On-Line Rapid Palliation Using Helical Tomotherapy: A Prospective Feasibility Study," Radiotherapy and Oncology, Vol. 87, No. 1, 2008, pp. 116-118. doi:10.1016/j.radonc.2008.01.017

[12] R. Samant, L. Gerig, L. Montgomery, R. Macrae, et al., "High-Technology Palliative Radiotherapy Using ImageGuided Intensity-Modulated Radiotherapy,” Clinical Oncology (Royal College of Radiologists), Vol. 20, No. 9, 2008, pp. 718-720. doi:10.1016/j.clon.2008.08.002

[13] R. Samant, L. Gerig, L. Montgomery, R. Macrae, et al., "The Emerging Role of IG-IMRT for Palliative Radiotherapy: A Single-Institution Experience,” Current Oncology, Vol. 16, No. 3, 2009, pp. 40-45.

[14] I. Lee, et al., "Early Clinical Experience and Outcome of Helical Tomotherapy for Multiple Metastatic Lesions,” International Journal of Radiation Oncology*Biology* Physics, Vol. 73, No. 5, 2009, pp. 1517-1524.

[15] S. Ryu, et al., "Pain Control by Image-Guided Radiosurgery for Solitary Spinal Metastasis,” Journal of Pain and Symptom Management, Vol. 35, No. 3, 2008, pp. 292-298. doi:10.1016/j.jpainsymman.2007.04.020

[16] J. Sheehan, et al., "Radiosurgery in the Treatment of Spinal Metastases: Tumour Control, Survival, and Quality of Life after Helical Tomotherapy,” Neurosurgery, Vol. 65, No. 6, 2009, pp. 1052-1062. doi:10.1227/01.NEU.0000359315.20268.73

[17] S. Lutz, S. S. Lo, E. Chow, A. Sahgal and P. Hoskin, "Radiotherapy for Metastatic Bone Disease: Current Standards and Future Prospectus," Expert Review of Anticancer Therapy, Vol. 10, No. 5, 2010, pp. 683-695. doi:10.1586/era.10.32

[18] J. Baisden, et al., "Helical Tomotherapy in the Treatment of Central Nervous System Metastasis," Neurosurgery Focus, Vol. 22, No. 3, 2007, pp. 1-6. doi:10.3171/foc.2007.22.3.9 